\title{
POLYPROPYLENE MESH IMPLANT AND A-PRF MEMBRANE FOR RECONSTRUCTION OF THE TRAUMATIC DEFECTS OF THE LATERAL AND BACK WALL OF THE MAXILLARY SINUS
}

\author{
Vladyslav Malanchuk \\ Department of Oral and Maxillofacial Surgery \\ National Medical University \\ 1 Zoologichna str., Kyiv, Ukraine, 03179 \\ malanchuk_v_a@ukr.net \\ Vadym Iefysko \\ Department of Oral and Maxillofacial Surgery \\ National Medical University \\ 1 Zoologichna str., Kyiv, Ukraine, 03179 \\ e_vadim@ukr.net \\ Iryna Logvynenko \\ Department of Oral and Maxillofacial Surgery \\ National Medical University \\ 1 Zoologichna str., Kyiv, Ukraine, 03179 \\ irina-mb@ukr.net \\ Natalia Iefysko \\ Department of Oral and Maxillofacial Surgery \\ National Medical University \\ 1 Zoologichna str., Kyiv, Ukraine, 03179 \\ evvadim@ukr.net
}

\begin{abstract}
Background: The aim of this study was to investigate the possible use of Polypropylene mesh implant and A-PRF membrane for covering the defects of the lateral and back wall of the maxillary sinus after its traumatic damages.

Materials: non-resorbable Polypropylene mesh implant was used in 10 patients as a barrier membrane for prevention of adipose tissue herniation into maxillary sinus.

Method: 3-D CT was used for assessment of bone fragments position and volumetric parameters of the maxillary sinus before and after surgical repair.

Result: Postoperative 3-D CT after 2 months demonstrated effective reestablishment of anatomical configuration of sinus and its volume, absence of pathological inflammatory changes of the sinus mucosa.

Conclusion: Polypropylene mesh implant provides effective barrier between soft tissues and maxillary sinus, due to its strength characteristic and biocompatibility.

Keywords: midfacial trauma, barrier membranes, A-PRF membrane, maxillary sinus walls defects.

\section{Introduction}

Traumatic injuries of the maxillo-facial area are actual medical problem; they take important place between other kinds of pathologies. Frequency of the zygomatic complex fractures reaches 16-5\% between other facial bones fractures; they took second place by frequency after fractures of the mandible $[1,2]$. Traumatic displacement of the zygomatic bone leads to crash of the thin walls of the maxillary sinus, haemosinus formation, and adipose tissue herniation with sinus volume decreasing [2-4]. Pathological posttraumatic changes alter function of the maxillary sinus mucosa drainage that often leads to inflammatory complications $[3,5,6]$.
\end{abstract}


Treatment of the fractures of the zygopmatic complex should be directed on reposition of the bone fragments, restoration of bone structures integrity, natural sinus drainage and aeration. For that purpose sanitation procedures have to be performed, such as sinus revision, debris and bone fragments removal. In cases of severe fragmentation of sinus walls it is important to provide reconstruction of lost bony structures and provide support for soft tissues and at the same time to prevent herniation of adipose tissue into the sinus.

Different reconstructive materials are used for reconstruction of maxillary sinus walls - titanium meshes, 1-polylactide plates, bone autotransplants and others. More often the reconstructive procedures are used in case of orbital floor defects and more rarely in case of defects of anterior sinus wall [9-11]. Disadvantage of those materials are difficulties of fixation on lateral and back wall of the maxillary sinus, and lack of flexibility.

Lately broad application of polypropylene meshes is observed in practice of surgical treatment of hernias, anterior abdominal wall plasty, internal organs fixation. Mesh endoprothesis are flexible, elastic, stable for action of tissue fluids and blood, provide ingrown of vessels and integration with surrounding tissues. They have no cancerogenic effect on tissues. After healing process connective tissue barrier appear, that is a positive feature of polypropylene mesh [7, 8].

Reconstruction of the lateral and back wall of the maxillary sinus is usually underestimated while treatment of zygomatic fractures.

Retrospective analysis of CT scans of patients with zygomatic complex fractures with tuber maxilla fragmentation and intraoperative observations revealed that herniation of soft tissues originates especially from the defect of lateral and back wall of maxillary sinus.

That is why in our clinical practice we pay attention not only on reestablishment of the zygomatic bone position, but also on normalization of maxillary sinus function [4-6]. Our treatment protocol includes revision of the maxillary sinus, bone fragments and clouts evacuation, reconstruction of the lateral and back wall of the maxillary sinus [7]. Polypropylene mesh was used as barrier membrane. Reconstruction procedures were enhanced by the usage of A-PRF membrane for stimulation of regenerative processes [8-10].

\section{Aim}

To estimate the effectiveness of usages of the Polypropylene mesh implant and A-PRF membrane for covering the defects of the lateral and back wall of the maxillary sinus for reestablishment of maxillary sinus volume and mucosa drainage function after treatment of zygomatic complex fractures.

\section{Materials and methods}

10 patients with zygomatic complex fractures were observed, 3-D CT scanning was performed. That patients group involved cases with comminuted fractures of the lateral and side wall of the maxillary sinus, displacement of bone fragments into the sinus, haemosinus that required surgical sinus revision. Postoperative 3-D CT was performed after two months, reestablishment of anatomical configuration of sinus and its volume, absence of pathological inflammatory changes of the sinus mucosa were estimated.

\section{Clinical case}

Patient B., 51 y. o., case history № 12985 was hospitalized with diagnosis: closed head injury, brain concussion, left side traumatic zygomatic complex fracture with fragments displacement, haemosinus. Trauma was 3 days ago. Treatment plan: reposition and osteosyntesis of the left zygomatic complex, reconstruction of the lateral and back wall of the maxillary sinus by Polypropylene mesh implant and A-PRF membrane. Venous blood in quantity of $30 \mathrm{ml}$ was harvested before operation into sterile vacuum tubes and centrifugation performed on regimen of $1500 \mathrm{rpm}$ during 10 min. Received fibrin clots were enriched by platelets by A-PRF methodic. Fibrin membranes than were formed (Fig. 1)

A-PRF contains grows factors: PDGF (platelet grows factor), TGF- $\beta$ (transformative grows factor), IGF (insulin-like grows factor), EGF (epithelium grows factor) and VEGF (endothelial ves- 
sels grows factor), that are key elements for wounds healing. By the dates of different researches $[5,6]$, all grows factors released slowly during 7-10 days from A-PRF and improve regeneration of soft tissues and bones.

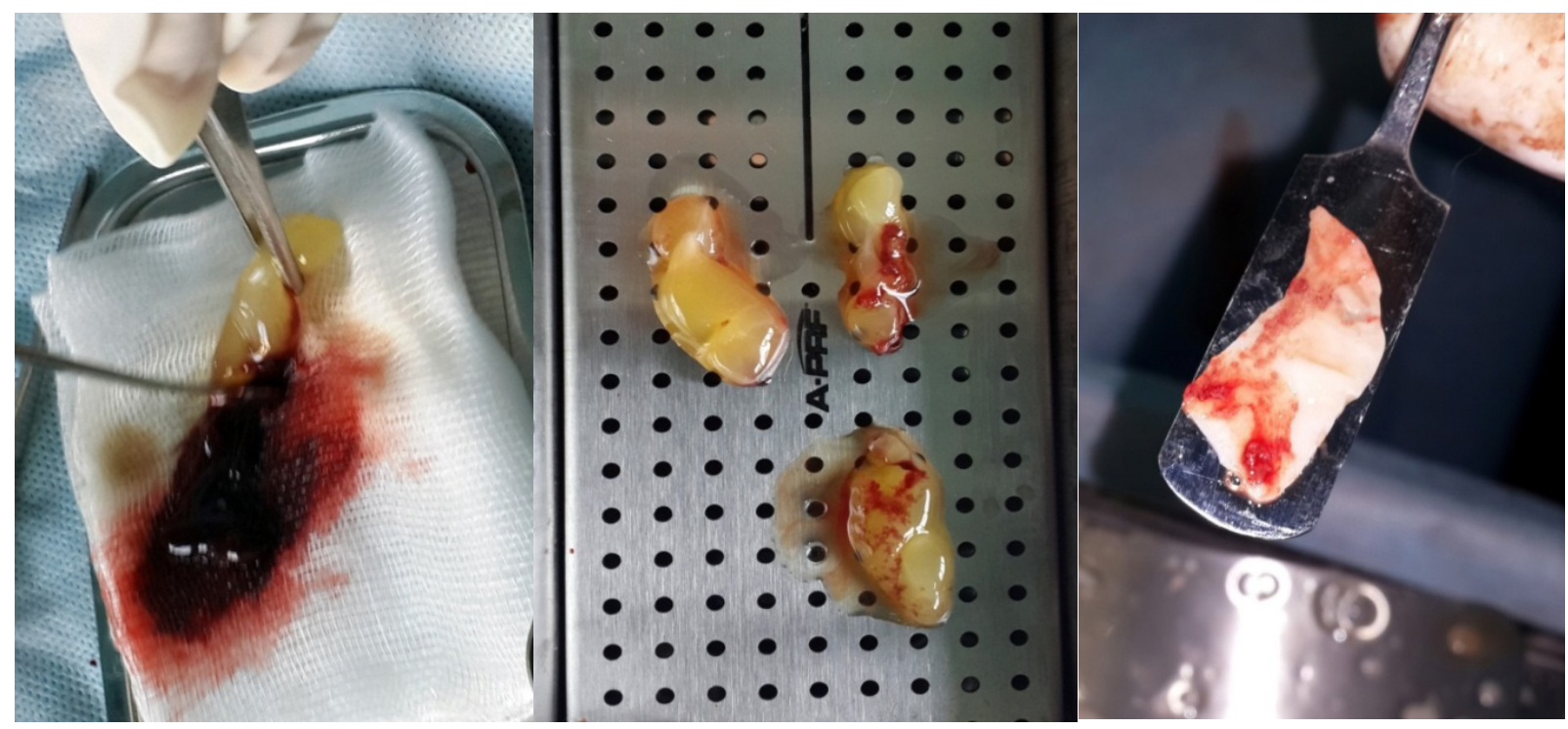

Fig. 1. Stages of fibrin membrane preparation

Operation was performed under general anesthesia. Intraoral vestibular access was used for opening fracture sides - zygomatico-alveolar ridge, anterior, lateral and posterior walls of maxillary sinus. Revision of maxillary sinus was performed through the defect of anterior wall after small bone fragments were removed. Sinus was cleaned from blood clots and free bone fragments. Intraoral reposition of zygomatic bone and fixation by titanium plates on the zygomatico-alveolar buttress was performed.

Adipose tissue of the cheek prolapsed into the sinus through the defects of lateral and posterior walls (Fig. 2)

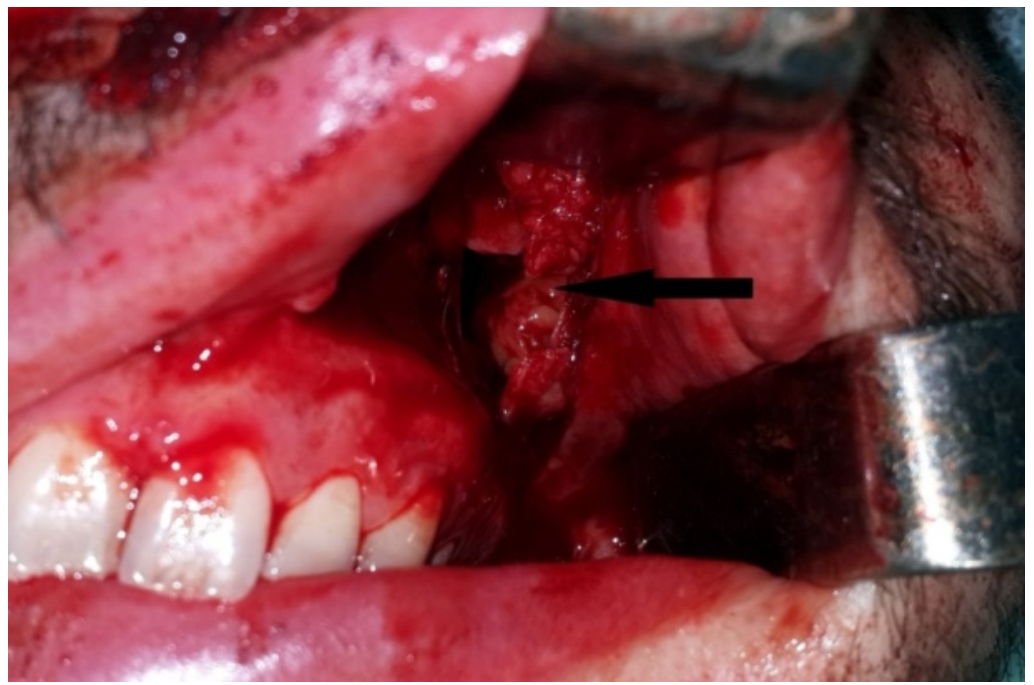

Fig. 2. Adipose tissue of the cheek that prolapses into the sinus

Non-resorbable Polypropylene mesh was used as a barrier membrane and positioned over bone defects vestibular between stable bone fragments and adipose tissue (Fig. 3). 


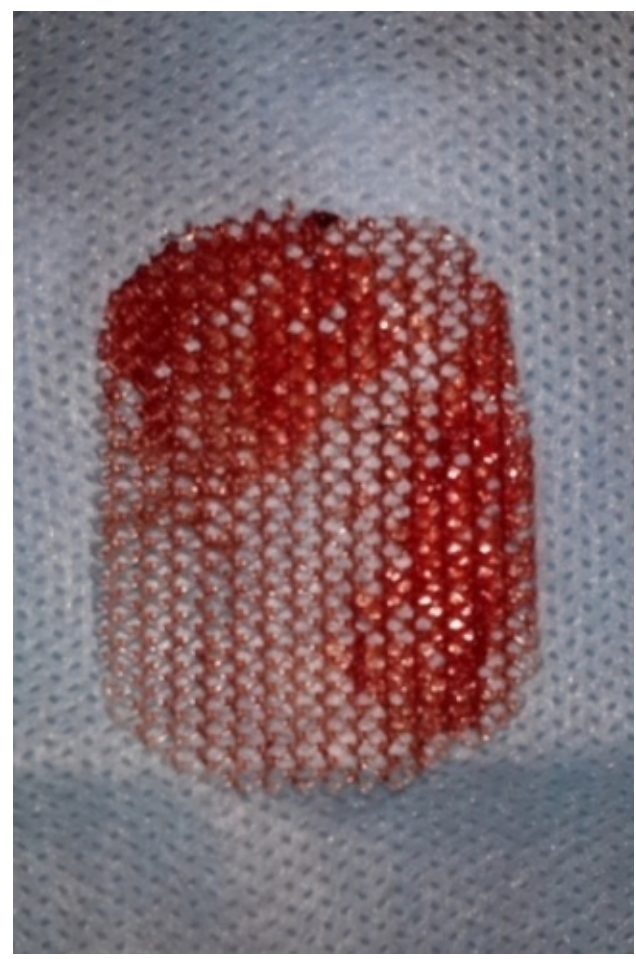

Fig. 3. Nonresorbable Polypropylene mesh

Usage of polypropylene mesh allowed reaching few purposes: provided secure barrier for soft tissues by sufficient rigidity of the mesh (Fig. 4); restoration of anatomical contour of maxillary walls and primary volume of the sinus.

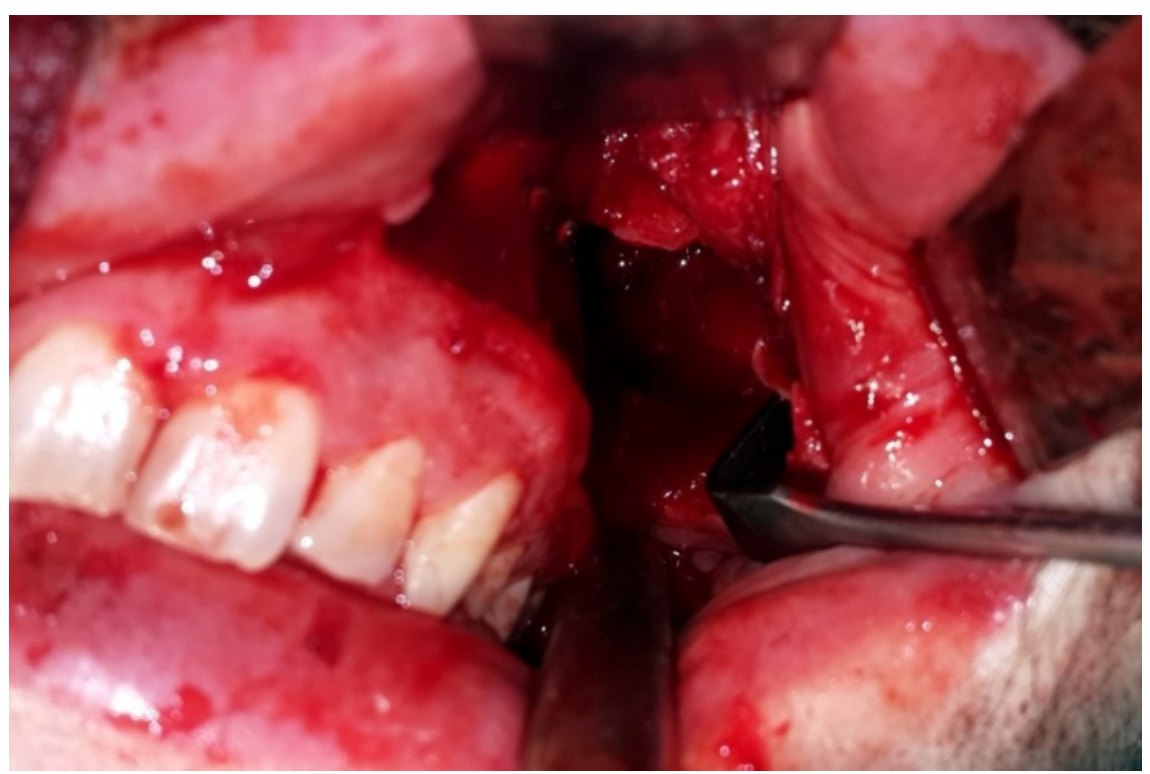

Fig. 4. Polypropylene mesh provided secure barrier for soft tissues

Sinus surface of the mesh was covered by fibrin membranes A-PRF to enhance regenerative processes - bone healing and sinus mucosa regeneration (Fig. 5).

Catheter was used during 3 days for irrigation of the sinus by antiseptic solutions (Fig. 6). 


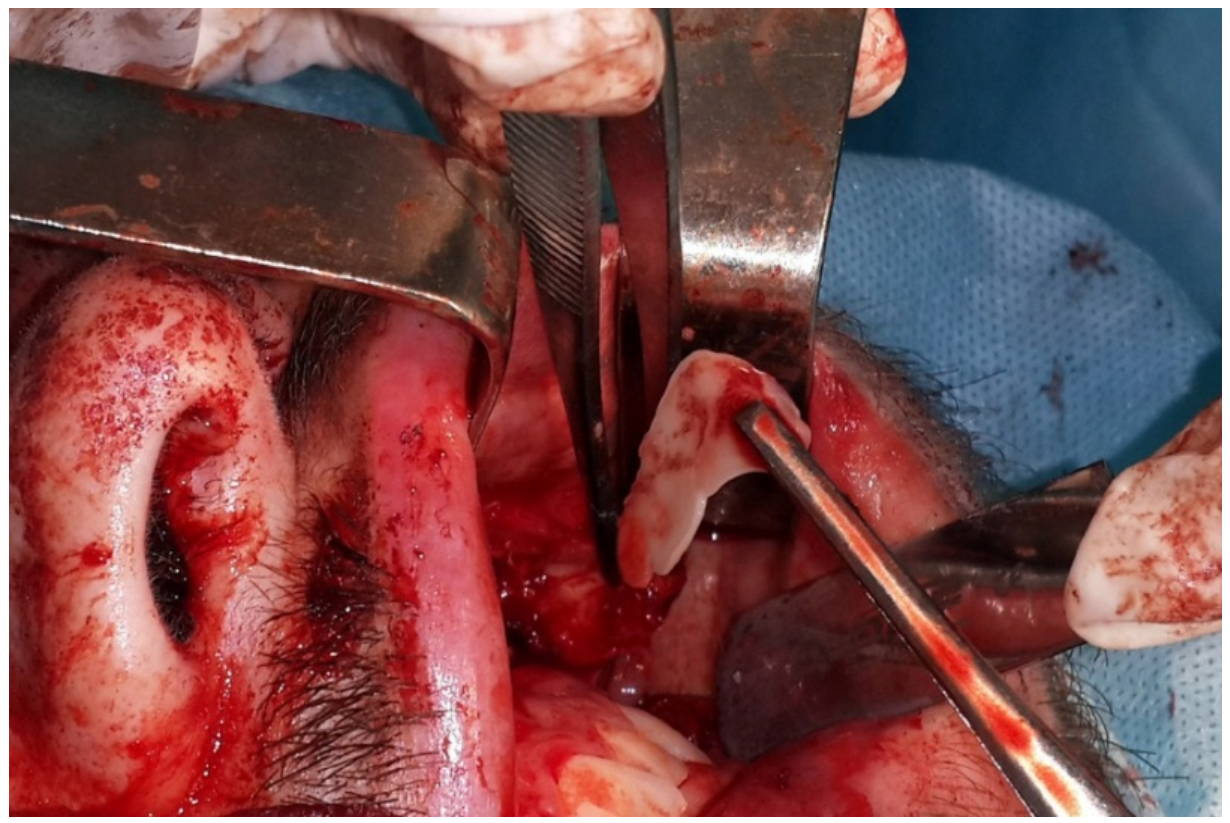

Fig. 5. Sinus surface of the mesh was covered by fibrin membranes

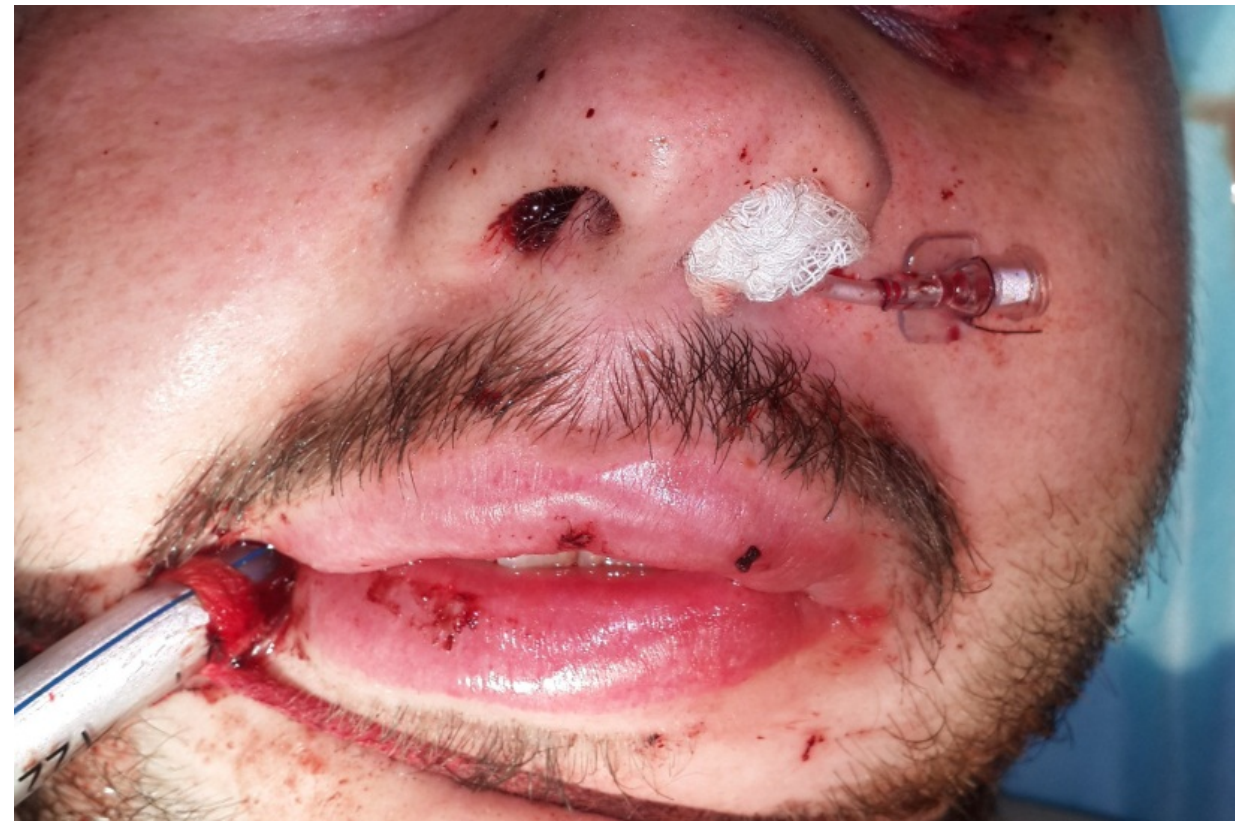

Fig. 6. Catheter placed via medial sinus wall for postoperative sinus irrigation

Preoperative 3-D CT evaluation reveals fragmentation of lateral and back wall of maxillary sinus with displacement of the fragments into sinus cavity and cause haemosinus. If that situation will be underestimated and not treated, posttraumatic sinusitis could develop. In our treatment algorithm - sanitation of maxillary sinus is performed after reposition and fixation of zygomatic bone fragments.

3-D CT control is usually performed after 2-3 month after operation. In these terms bone fragments position, the state of the maxillary sinus mucous and sinus volume could be estimated. Postoperative 3-D CT of presented case two month after operation demonstrates effective reestablishment of anatomical configuration of sinus and its volume, absence of pathological inflammatory changes of the sinus mucosa due to optimization of healing process. 

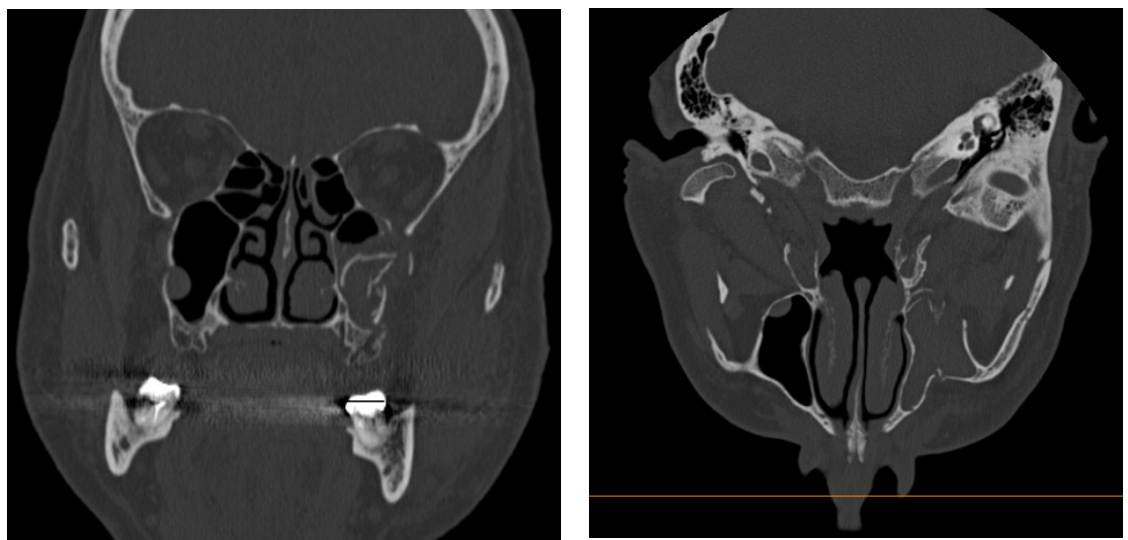

Fig. 7. 3-D CT reveals traumatic crash of the lateral and back walls of the maxillary sinus after zygomatic fracture
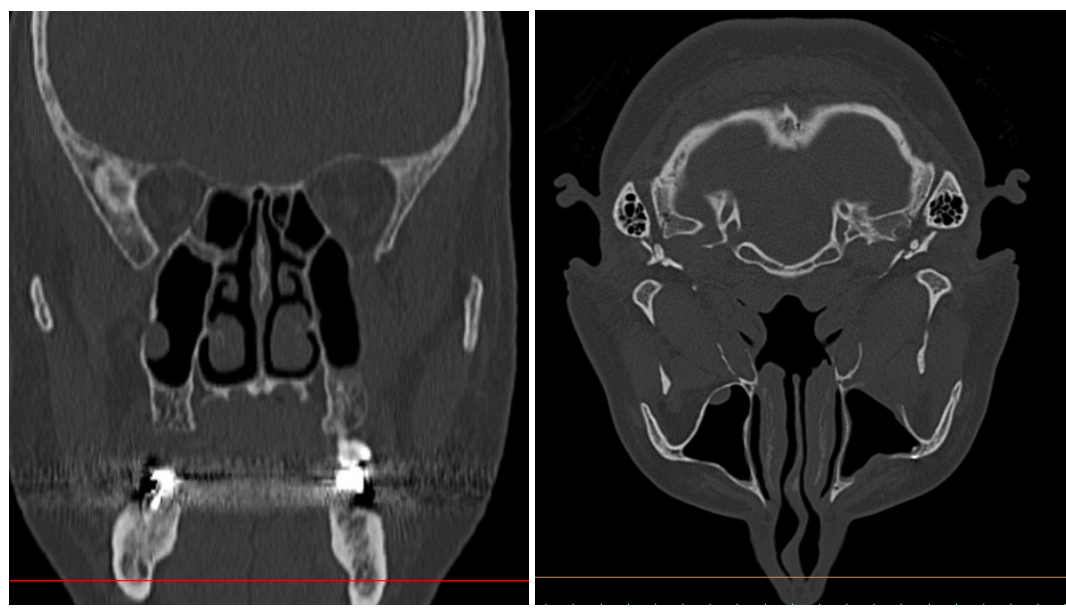

Fig. 8. Postoperative 3-D CT after two month demonstrates effective reestablishment of anatomical configuration of sinus and its volume, absence of pathological inflammatory changes of the sinus mucosa.

\section{Results}

Before the introduction of polypropylene mesh in practice we have studied and analyzed the experience of using this material in other areas of medicine. The advantages of the use of polypropylene mesh include:

1) simple modeling material in the area of the defect;

2) no need for additional fixing elements. Mesh is fixed by its own adhesion to tissues between the bony walls and soft tissues;

3) no need to remove the implant;

4) the formation of resistant barrier between the soft tissues and maxillary sinus lumen;

5) does not cause atrophy of the surrounding soft tissues.

Disadvantages include:

1) the elasticity of the material makes it impossible to use for insulation cavities with high pressure surrounding soft tissues. So, at the bottom of the defect orbit grid cannot be used as a mono material, as the pressure of the eyeball and orbital adipose tissue into the lumen of the net chasing maxillary sinus.

2) does not promote bone formation in the area of the defect;

3) is a foreign body;

4) not resorbed. 
Polypropylene non-resorbable mesh was used for covering the defects of lateral and back wall of the maxillary sinus in 10 cases of zygomatic complex fractures. Postoperative 3-D CT after 2 months demonstrated effective reestablishment of anatomical configuration of sinus and its volume, absence of pathological inflammatory changes of the sinus mucosa (Fig. 7, 8).

\section{Conclusion}

1. Polypropylene non-resorbable mesh is recommended for covering the posttraumatic defects of lateral and back wall of the maxillary sinus.

2. Polypropylene non-resorbable mesh provides effective barrier for soft tissues. High biocompatibility of the material minimizes risk of inflammatory complications.

3. Usage of the A-PRF membrane improves regeneration of soft tissues and bones.

4. Combination of usage of artificial and biologic membranes enhances the results of anatomical reconstruction and optimization of healing processes.

\section{References}

[1] Li, Y. S., Tian, W. D., Li, S. W., Liu, L. (2006). Retrospective analysis of 3,958 patients with facial injuries. Zhonghua Kou Qiang Yi Xue Za Zhi, 41 (7), 385-387.

[2] Pathak, H., Mohanty, S., Urs, A. B., Dabas, J. (2015). Treatment of Oral Mucosal Lesions by Scalpel Excision and Platelet-Rich Fibrin Membrane Grafting: A Review of 26 Sites. Journal of Oral and Maxillofacial Surgery, 73 (9), 1865-1874. doi: 10.1016/j.joms.2015.03.041

[3] Ruzin, G. P., Poberezhnik, G. A. (2011). The impact fracture of the zygomatic bones on the state of the mucosa of the maxillary sinus. Ukr. stomat. Almanac, 4, 17.

[4] Medvedev, Y., Shamanaeva, L. S., Soloviev, A. A. (2011). Reconstruction of the walls of the maxillary sinus in fractures of the middle zone of the facial skull. Russian Rhinology, 42, 37.

[5] Kalashnikov, E. H., Sivolapov, K. A. (2008). The results of the application of endoscopy in the treatment of patients with old fractures, deformities and defects of the lateral midface department. Sib. med. obozrenie, 6, 88-89.

[6] Edranov, S. S. (2005). Structural changes in the mucosa of the maxillary sinus when mechanical trauma. Vladivostok, 22.

[7] Baburin, A. B. (2014). Vibor plastic technique for inguinal grizhah in young men. Nizhny Novgorod, 96.

[8] Flament, J. B., Avisse, C., Palot, J. P., Delattre, J. F. (2000). Complications in incisional hernia repairs by the placement of retromuscular prostheses. Hernia, 4 (S1), S25-S29. doi: 10.1007/bf01387179

[9] Kabanov, A. A., Kabanov, S. A., Bogdan, N. Y., Chernin, T. N. (2013). Plastic front wall of the maxillary sinus. Bulletin VSMU, 12 (3), 11.

[10] Kuttenberger, J. J., Hardt, N., Treumann, T. C. (2004). CT-kontrollierte Sptergebnisse nach Defektdeckung im Kieferhhlenbereich mit dem Mikrotitangitter. Mund-, Kiefer- Und Gesichtschirurgie, 8 (6), 330-336. doi: 10.1007/s10006-004-0579-z

[11] Pokrovskaya, E. M. (2015). The modern transplants for the restoration of bone defects in rhinology. Vestnik Otorinolaringologii, 80 (4), 22-26. doi: 10.17116/otorino201580422-26

[12] Bains, V., Gupta, V., Jhingran, R., Mathur, A., Singh, G. (2015). Evaluation of intrabony defects treated with platelet-rich fibrin or autogenous bone graft: A comparative analysis. European Journal of Dentistry, 9 (1), 100. doi: 10.4103/1305-7456.149653

[13] Yuanzheng, C., Yan, G., Ting, L., Yanjie, F., Peng, W., Nan, B. (2015). Enhancement of the Repair of Dog Alveolar Cleft by an Autologous Iliac Bone, Bone Marrow-Derived Mesenchymal Stem Cell, and Platelet-Rich Fibrin Mixture. Plastic and Reconstructive Surgery, 135 (5), 1405-1412. doi: 10.1097/ prs.0000000000001166 